\title{
Síndrome de Achenbach: apresentação de um relato de caso e revisão de literatura
}

\author{
Achenbach Syndrome: presentation of a case report and review of the literature \\ Síndrome de Achenbach: presentación de un caso clínico y revisión de la literatura
}

Recebido: 21/08/2021 | Revisado: 28/08/2021 | Aceito: 08/09/2021 | Publicado: 11/09/2021

\author{
Jéssica Lie Utiamada \\ ORCID: https://orcid.org/0000-0003-3981-4311 \\ Universidade do Planalto Catarinense, Brasil \\ E-mail: jeeeutiamada@gmail.com \\ Pâmela Mayara da Silva Cassemiro \\ ORCID: https://orcid.org/0000-0003-3252-2594 \\ Universidade do Planalto Catarinense, Brasil \\ E-mail: pam.casse@hotmail.com \\ Rafael Cristiano Brandl \\ ORCID: https://orcid.org/0000-0002-6475-8318 \\ Universidade do Planalto Catarinense, Brasil \\ E-mail: rafaelbrand138@gmail.com \\ Bertha Aparecida Davet \\ ORCID: https://orcid.org/0000-0002-1235-5082 \\ Universidade do Planalto Catatinense, Brasil \\ E-mail: berthadavet@yahoo.com.br \\ João Pedro Pereira da Cunha \\ ORCID: https://orcid.org/0000-0002-3716-9921 \\ Universidade do Planalto Catarinense, Brasil \\ E-mail: joaopedro.ref@gmail.com
}

\begin{abstract}
Resumo
O objetivo deste trabalho é descrever um relato de caso de um paciente adulto com diagnóstico prévio de uma doença autoimune. Configura-se como estudo do tipo relato de caso clínico por meio de coleta direta dos dados do paciente, descrevendo os acontecimentos de forma cronológica, visando o melhor entendimento do quadro clínico e ações médicas realizadas. A paciente procurou atendimento na cidade de Lages no dia 09/10/2019, devido a uma queixa de alteração em coloração de mãos, de início há 4 meses, persistindo por 4 horas. Sem achados de significância clínica em exame físico. Durante investigação ambulatorial fora realizados exames laboratoriais e Doppler arterial de membros superiores, sem evidência de vasculopatia, doenças hematológicas ou outras patologias. Após exclusão de demais doenças a Síndrome de Achenbach tornou-se a de maior probabilidade. Devido a evolução favorável dessa patologia, fora prescrito alguns medicamentos para melhora sintomática de paciente e solicitação de exames para reavaliação de quadro em 6 meses. Desta forma conclui-se que a síndrome de Achenbach configura-se como uma patologia complexa, uma vez que sua etiologia e fisiopatologia não são bem definidas, gerando dificuldade na realização de diagnóstico dessa entidade pouco conhecida.
\end{abstract}

Palavras-chave: Síndrome de Achenbach; Hematoma nas extremidades; Cianose.

\begin{abstract}
The objective of this work is to describe a case report of an adult patient whit a previous diagnosis of a autoimmune disease. Better understanding of the clinical picture and medical actions performed. The patient sought care in the city of Lages on 10/09/2019, due to a complaint of changes in hand color, beginning 4 months ago, persisting for 4 hours. No fingings of clinical significance on physical examination. During outpatient investigation, laboratory tests and arterial Doppler of the upper limbs were performed, without evidence of vasculopathy, hematological diseases or other pathologies. After excluding other diseases, Achenbach's Syndrome became the most likely one. Due to the favorable evolution of this pathology, some medications had been prescribed for the patient's symptomatic improvement and requests for exames to reassess the condition in 6 months. Thus, it is concluded that Achenbach's syndrome is configured as a complex pathology, since its etiology and pathophysiology are not well defined, causing difficulty in making the diagnosis of this little-known entity.
\end{abstract}

Keywords: Achenbach Syndrome; Hematoma in the extremities; Cyanosis.

\section{Resumen}

El objetivo de este trabajo es describir un reporte de caso de un paciente adulto con diagnóstico previo de una efermedad autoinmune. Se configura como un estudio de reporte de caso clínico mediante la recolección directa de datos del paciente, describiendo los eventos de forma cronológica, con el objetivo en una mejor comprensión del cuadro clínico y las acciones médicas realizadas. El paciente buscó atención en la ciudad de Lages el 09/20/2019, debido a una queja 
de cambios en el color de la mano, iniciada hace 4 meses, persistiendo durante 4 horas. No hay hallazgos de importancia clínica en la expliración física. Durante la investigación ambulatoria, se realizaron pruebas de laboratory y Doppler arterial de miembros superiores, sin evidencia de vasculopatía, enfermedades hematológicas u outras patologías. Después de excluir otras efermedades, el Síndrome de Achenbach se convirtió en el más probable, debido a la evolución favorable de esta patología, se prescribieron algunos medicamentos para la mejoría sintomática del paciente y se solicitaron pruebas para reevaluar la condición en el plazo de 6 meses. Así, se concluy el Síndrome de Achenbach se configura como una patología compleja, ua que su etiología y fisiopatología no están bien defínidas, lo que dificulta el diagnóstico de esta entidad poco conocida.

Palabras clave: Síndrome de Achenbach; Hematoma en las extremidades; Cianosis.

\section{Introdução}

A Síndrome de Achenbach, também conhecida como hematoma paroxístico do dedo ou dedo azul idiopático agudo é uma entidade caracterizada pelo aparecimento de equimoses ou hematomas na face palmar, plantar, quirodáctilos ou pododáctilos. Tal condição pode ser secundária a um trauma, em certos casos. É uma síndrome rara, de caráter benigno e de curso autolimitado, a qual pode ser acompanhada de dor, dormência no local afetado, edema com limitação de movimento e uma sensação de frio local. Há remissão na maioria das vezes de forma espontânea, dentro de poucos dias a semanas, sem sequelas permanentes, apresentando um prognóstico favorável (Jaimes, Chinome \& Molina-Franky 2019).

Sua patogênese ainda não está completamente estabelecida, mas como os sintomas são tipicamente desencadeados por atividades cotidianas e aumento da fragilidade vascular local, possivelmente se relaciona com a idade do paciente ou pequenos traumas, causando micro-hemorragias capilares. Entretanto, muitos pacientes desenvolvem a doença sem um fator predisponente. Outra explicação seria pela redução de fluxo sanguíneo devido ao vasoespasmo ou compressão por hematomas locais. O sangramento subdérmico geralmente cessa espontaneamente ou após uma pressão local ser aplicada, e a coloração desta alteração desaparece sem passar pelos típicos estágios de reabsorção de equimose. Além disso, as falanges distais e o leito ungueal são preservados, ao contrário do que se observa nas doenças isquêmicas (Godoy \& Tabares, 2019; Jaimes, Chinome \& Molina-Franky, 2019).

O quadro clínico pode ser totalmente assintomático, mas quando apresenta sintomas, ocorre inicialmente com uma sensação de queimação ou desconforto em um ou mais dedos, evoluindo para hematomas associado a edema, parestesia, e por vezes limitação da mobilidade. Seu curso é benigno e os sintomas têm resolução espontânea em média de uma semana. Seu diagnóstico baseia-se na história clínica, associada a exames complementares que visam excluir outros diagnósticos diferenciais (Godoy \& Tabares, 2019; Jaimes, Chinome \& Molina-Franky, 2019).

Existem diferentes condições patológicas que se apresentam com mudanças na coloração das extremidades e reconhecêlas é de grande interesse médico, pois alguns envolvem uma doença subjacente potencialmente grave (Godoy \& Tabares, 2019).

Com isso, o objetivo deste trabalho é relatar a síndrome de Achenbach, com relação às suas possíveis etiologias, seu quadro clínico, diagnóstico e o manejo dos pacientes afetados.

\section{Metodologia}

O artigo, segundo Pereira A.S. et al. (2018), corresponde a um estudo de caso clínico, com caráter qualitativo, realizado de forma retrospectiva e observacional. Para a revisão bibliográfica foram utilizadas as seguintes palavras-chave: Síndrome de Achenbach; Hematoma de extremidades; Cianose. Sendo pesquisado em artigos e revisões bibliográficas, publicadas no período de 2005 e 2020. O trabalho desenvolvido por meio da avaliação de prontuário médico, sob o consentimento e assinatura do Termo de Consentimento Livre e Esclarecido, segundo Resolução 466/2012 CNS/CONEP. 


\section{Caso Clínico}

Paciente do sexo feminino, 48 anos, com diagnóstico prévio de tireoidite de Hashimoto, em uso contínuo de Levotiroxina 175 mcg e Colecalciferol 10.000 UI/semana, procura atendimento devido a alteração de coloração de mãos. Início do quadro há 4 meses, com crises repetidas, persistindo por mais de 4 horas, em caráter equimótico/hematomas, sem palidez ou hiperemia associada (Figura 1). Não havia relato do frio como desencadeante das crises. Realizada investigação inicial com hemograma, CPK, creatinina, VHS, PCR, eletroforese de proteínas e dosagem de C3, C4 e CH50, sendo todos com resultados normais. FAN, anti-DNA, anti-Ro anti-La, Fator reumatoide, ANCA C e ANCA P negativos. Sorologias para HIV, Hepatite B, Hepatite C e VDRL negativos. Doppler arterial de membros superiores sem alterações sugestivas de doença vascular. Após avaliação de história clínica e de exames complementares, excluindo os principais diagnósticos diferenciais, fecha-se o diagnóstico da síndrome de Achenbach. Realizadas orientações sobre a benignidade do quadro e sugerido tratamento sintomático. Solicitou-se realização de novos exames complementares em 6 meses para reavaliação de quadro.

Figura 1 - Comprometimento mais significativo de $4^{\circ}$ quirodáctilo de mão direita.

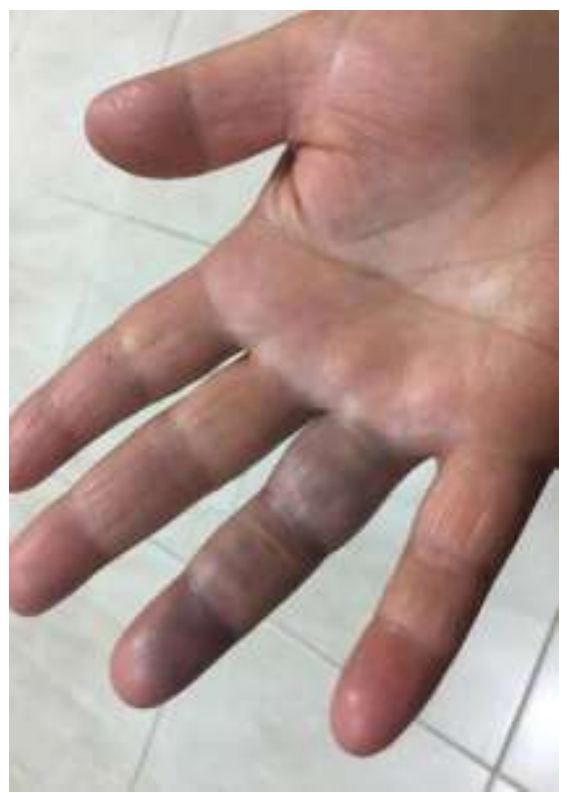

Fonte: Fotografia retirada em atendimento realizado na clínica AMA (Lages - SC) 
Figura 2 - Comprometimento mais significativo de $2^{\circ}$ quirodáctilo de mão direita.

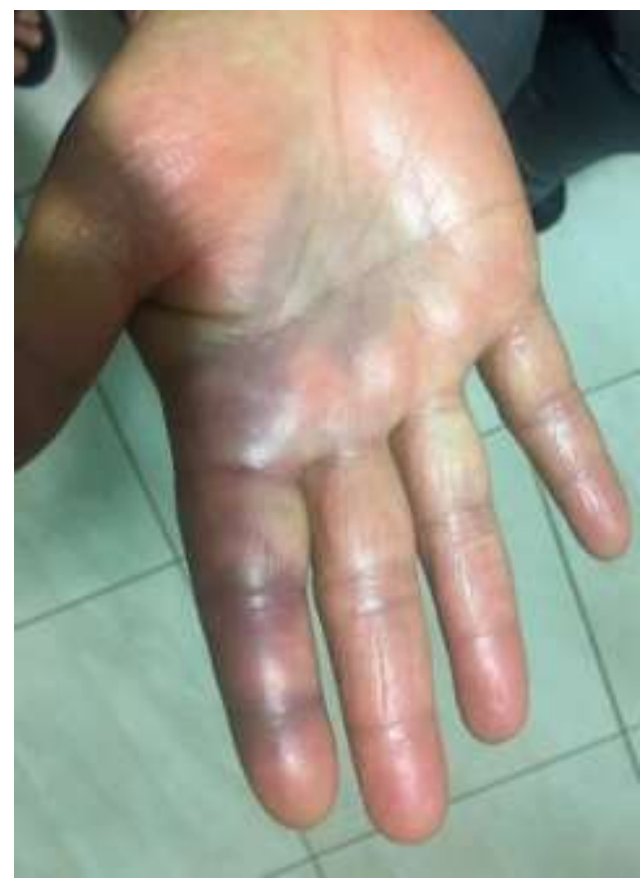

Fonte: Fotografia retirada em atendimento realizado na clínica AMA (Lages - SC)

\section{Discussão}

A Síndrome de Achenbach foi relatada pelo médico alemão Walter Achenbach, em 1956. Em seu trabalho original W. Achenbach, descreveu casos de 6 mulheres que sofreram crises de dor em região palmar dos dedos, de caráter agudo e recorrente, seguido de mudanças na coloração das regiões afetadas (Achenbach, 1957; Ribeiro, et al., 2019; Jaimes, Chinome \& MolinaFranky, 2019).

Essa entidade clínica continua ainda pouco conhecida, com poucos relatos na literatura. Uma revisão de estudo sistemática, realizado em 2016, identificou 12 relatos de caso, a nível mundial, concluindo que a idade média de apresentação fora aos 49,5 anos de idade, predominantemente em mulheres (91\% dos casos), associado a dor e edema em 58\% dos pacientes e parestesias/prurido em 25\% (Kordzadeh, et al., 2016).

A fisiopatologia dessa condição não está bem elucidada, mas alguns autores correlacionam a patogênese com a fragilidade capilar, consequência da idade, em associação à fatores genéticos, considerando a observação de vários casos da doença em membros de uma mesma família. Outra justificativa para essa condição pode ser a redução do fluxo sanguíneo devido à ocorrência de vasosepasmo (Cohen, 2018; Yamada, 2018; Mishra, Jain, Sen \& Majumdar, 2015; Frerix, Richter, Müller-Ladner \& Hermann, 2015).

O quadro clínico envolve episódios súbitos de edema e dor, em um ou mais dedos das mãos ou pés, que precede em minutos à horas a alteração de coloração das áreas afetadas, com surgimento de hematoma, havendo predomínio da região palmar das falanges proximais, de acordo com a literatura, não há traumas associados. As falanges distais e os leitos ungueais são geralmente poupados, fator que diferencia a síndrome de Achenbach das doenças isquêmicas. Podem também estar presentes quadros de parestesia, limitação da movimentação e sensação de frio nos dedos afetados. Em poucos dias a semanas, há o desaparecimento dos sintomas, não deixando sequelas, evidenciando, dessa forma, o caráter benigno e de bom prognóstico da doença (Godoy \& Tabares, 2019; Jaimes, Chinome \& Molina-Franky, 2019; Carpentier, et al., 2016).

O diagnóstico da síndrome de Achenbach é de exclusão, portanto é realizado por meio de história clínica e exame físico compatíveis, além de afastar outras condições que podem cursar com sinais e sintomas semelhantes. Podem ser realizados exames 
como hemograma, coagulograma, radiologia convencional, análise de autoanticorpos, doppler e arteriografia dos membros. Todos esses exames complementares podem estar com resultados dentro do limite de normalidade. A biópsia pode apresentar sinais inespecíficos, como sangramento leve e alterações inflamatórias (Godoy \& Tabares, 2019; Ribeiro, 2019).

Os diagnósticos diferenciais incluem isquemia do membro superior - por evolução aguda e sintomas semelhantes, como palidez, cianose, dor, edema e parestesias - fenômeno de Raynaud, tromboangeíte obliterante, acrocianose, vasculite, púrpura psicogênica e hematoma traumática (Godoy \& Tabares, 2019).

A Tromboangeíte obliterante, também conhecida como Síndrome de Buerger, é uma doença arterial obstrutiva periférica não aterosclerótica, que pode ser semelhante a isquemia aguda em membros superiores e à Síndrome de Achenbach. Todavia, a Tromboangeíte está associada ao tabagismo e sua evolução resulta em gangrena e ulceração dos dedos. Nessa doença, há dor e necrose em falanges distais com melhora significativa se houver cessação do uso de tabaco. A principal forma de diferenciação da Síndrome de Achenbach com a Tromboangeíte e isquemia de extremidades está na verificação de necrose e acometimento de falanges distais sem resolução espontânea (Rivera-Chavarría \& Brenes-Gutiérrez, 2016; Santana, et al., 2020; Watchorn, et al., 2017).

O fenômeno de Raynaud também deve ser considerado e apresenta caráter crônico e recorrente, com envolvimento de um ou mais dedos, apresentando variação da coloração com palidez e tons arroxeados. As crises ocorrem em decorrência de alterações de temperatura e melhora com o aquecimento das regiões acometidas (Wigley \& Flavahan, 2016).

A Acrocianose é uma condição que também deve ser considerada. Afeta igualmente o sexo feminino e masculino, com idade média de acometimento de 30 anos. A acrocianose é mais comum em indivíduos com baixo índice de massa corporal e é mais prevalente em locais com climas frios. Manifesta-se como cianose simétrica e indolor em mãos, dedos e rosto, sendo que pode ser precipitada por água fria. Não há fase de palidez e não há associação com úlcera ou necrose de falanges distais (Kurklinsky, Miller \& Rooke, 2011).

A Síndrome de Gardner-Diamond, também denominada púrpura psicogênica, pode simular a síndrome de Achenbach. Essa condição foi descrita pela primeira vez por Frank Gardner em 1955 e manifesta-se com placas eritematosas dolorosas que podem ser múltiplas ou únicas, que progridem para equimoses em 24 horas e que desaparecem em 7 a 10 dias sem deixar cicatrizes. Há maior acometimento do sexo feminino e diferente da síndrome de Achenbach pode afetar qualquer parte do corpo (mãos, pés, face e tronco) tendo também relação com transtornos de personalidade, náuseas, síncope ou sangramento (Estrada, et al., 2016; Karakas, et al., 2014).

A Eritromelalgia e eritema pérnio são consideradas diagnósticos diferenciais, pois podem evoluir com dor e edema dos dedos. No caso da eritromelalgia há a tríade de eritema, calor e dor nas extremidades que são deflagrados pela exposição ao calor. Essa condição afeta todas as faixas etárias e é três vezes mais comum em mulheres. O eritema pérnio é uma patologia inflamatória cutânea inflamatória com crises decorrentes da exposição ao frio. As lesões cessam espontaneamente em uma a três semanas, podendo haver recidivas (Godoy \& Tabares, 2016; Sanabria, et al., 2020).

As vasculites, por sua vez, devem ser investigadas e consistem em um grupo heterogêneo de doenças que se configuram pela inflamação dos vasos sanguíneos de diferentes calibres com consequente destruição das paredes vasculares podendo evoluir para sangramentos ou pela formação de trombos que podem originar isquemia da região afetada. O quadro clínico envolve febre, artralgia, mialgias, astenia e perda de peso. Devido à inespecificidade dos sintomas há dificuldade no diagnóstico dessas condições. Com a evolução do quadro pode haver sopros múltiplos, claudicação de extremidades, insuficiência aórtica e síncope e redução dos pulsos e da pressão arterial em um ou em ambos os membros superiores se houver acometimento de uma ou ambas as artérias subclávias (Lakdawala \& Fedeles, 2017; Gonçalves, 2019).

Por fim, ressalta-se a importância de tornar a Síndrome de Achenbach parte do conhecimento dos profissionais de saúde, 
pois devido a sua raridade há dificuldade na consideração dessa etiologia, já que existem diferentes condições patológicas que se apresentam com mudanças na coloração do extremidades. Reconhecer essas patologias e diferenciá-las é de grande interesse aos médicos, a fim de identificar e tratar aquelas com desfechos desfavoráveis e tranquilizar e oferecer suporte para os pacientes que apresentam condições de caráter benigno (Jaimes, Chinome \& Molina-Franky, 2019; Gonçalves, 2019).

\section{Conclusão}

A Síndrome de Achenbach configura-se como uma patologia médica complexa, uma vez que sua etiologia e fisiopatologia não são bem definidas, gerando dificuldade na realização de diagnóstico precoce e abordagem terapêutica específica. Os sintomas típicos apresentam remissão espontânea e não deixam sequelas, logo, o mais importante é a adequada exclusão de diagnósticos diferenciais com evoluções desfavoráveis.

Atualmente, sabe-se que existem poucos relatos conhecidos referente a esta patologia, sendo a maioria deles corresponde a casos clínicos e relatos de casos. Por este motivo, considerou-se importante a realização deste artigo, pela novidade do assunto, com escassa frequência de literatura brasileira e estrangeira, má suspensão por desconhecimento da síndrome.

Como trabalhos futuros, espera-se desenvolver mais estudos sobre a Síndrome de Achenbach, com uma fisiopatologia esclarecida, para melhor continuidade com o diagnóstico e abordagem terapêutica, evitando iatrogenias e/ou intervenções que não visam a melhora do paciente ou que não tratam sintomas em crise aguda.

\section{Referências}

Achenbach, W (1957). Ematomi parossistici della mano: Paroxysmal hematoma of the hand. Athena; 23(6):187-9.

Ahmed, Z., Elmallah, A., Elnagar, M., Dowdall, J., Barry, M. \& Sheehan, S. J (2018). Painful Blue FingerdAchenbach's Syndrome: Two Case Reports. EJVES Short Reports 40, 1e2. https://www.ejvesreports.com/article/S2405-6553(18)30022-7/pdf.

Carpentier P. H., Maricq H. R., Biro C., Jiguet M. \& Seinturier C (2016). Paroxysmal finger haematoma--a benign acrosyndrome occurring in middle-aged women. Vasa; 45(1):57-62. 10.1024/0301-1526/a000496

Cohen S. N. (2018). Personal observations of Achenbach syndrome. Clin Exp Dermatol; 43(8):933-4

Estrada J. S., Toquica F. E. R., Muñoz-Grajales C., Franco C. J. V., Hernández J. D. M. \& Peñaranda L. F. (2016) Síndrome de Gardner-Diamond como diagnóstico diferencial de lupus. Rev Colomb Reumatol; 23(3): 195- 199.

Frerix M., Richter K., Müller-Ladner U. \& Hermann W (2015). Achenbach's syndrome (paroxysmal finger hematoma) with capillaroscopic evidence of microhemorrhages. Arthritis Rheumatol; 67(4):1073. 10.1002/art.39003.

Godoy, A. \& Tabares, A. H (2019). Achenbach Syndrome (Paroxysmal Finger Hematoma). Vascular Medicine, 24(4) 361-366. 10.1177/1358863X19849627.

Godoy, A. \& Tabares, A. H (2019). Hematoma Paroxístico de Los Dedos (Síndrome de Achenbach). Rev. argent. dermatol; 100 (4): 135 - 144. http://www.scielo.org.ar/scielo.php?script=sci_arttext\&pid=S1851-300X2019000400111.

Godoy, A. \& Tabares, A. H (2016). Eritromelalgia. Experiencia Médica; 256(1): 32-34

Gonçalves, M. S. (2019). Vasculites: Desafio Diagnóstico e Terapêutico. Arq. Catarin Med.; 48(4):174-190 https://docs.bvsalud.org/biblioref/2020/01/1048 285/531-1838-4-rv.pdf

Jaimes, Y. A. P., Chinome, J. E. O \& Molina-Franky, J. (2019). Hematoma Digital Espontáneo, Síndrome de Achenbach. Revista de la Facultad de Ciencias Médicas de Córdoba; 76(4): 257-260. http://dx.doi.org/10.31053/1853.0605.v76.n4.24963.

Jimenez, P. R., Ocampo, M. I., Castaneda-Cardona, C. \& Rosselli, D (2017). Síndrome de Achenbach: presentación de un caso y revisión sistemática de la literatura. Rev. Colomb. Reumatol.; 2 4(4):230-236. https://www.researchgate.net/publication/320774317_Sindrome_de_Achenbach_presenta cion_de_un_caso_y_revision_sistematica_de_la_literatura.

Kampfen, S., Santa, D. D. \& Fusetti, C (2005). A Paintful Blue Thumb: A case of Achenbach`s Syndrome. EJVES Extra. 10.1016/j.ejvs.2005.07.009.

Karakaş, Z., Karaman, S., Avcı, B., Ünüvar, A., Öztürk, G., Anak, S., \& Devecioğlu, Ö. (2014). A disease difficult to diagnose: Gardner-Diamond syndrome accompanied by platelet dysfunction. Turk pediatri arsivi, 49(3), 250-253. https://doi.org/10.5152/tpa.2014.63

Kordzadeh A., Caine P. L., Jonas A., Rhodes K. M. \& Panayiotopolous Y. P. (2016). Is Achenbach's syndrome a surgical emergency? A systematic review. Eur J Trauma Emerg Surg; 42(4):439-443. 10.1007/s00068-015-0610-0. 
Kurklinsky A. K., Miller V. M. \& Rooke T. W (2011). Acrocyanosis: the Flying Dutchman. Vascular Medicine; 16(4):288-301. 10.1177/1358863x11398519

Lakdawala N. \& Fedeles F (2017). Vasculitis: Kids are not just little people. Clin Dermatol; 35(6):530-540. 10.1016/j.clindermatol.2017.08.004.

Mishra P., Jain A., Sen S. \& Majumdar B. (2015). Recurrent Bleeding and Bruising Over Little Finger: A Diagnostic Conundrum. Indian J Dermatol; 60(6): 632-633.

Ribeiro, F., Aveiro, M., Leal, M., Valente, T., \& Jesus, G. (2019). An Acute Blue Finger: A Case of Achenbach's Syndrome. European journal of case reports in internal medicine, 6(9), 001231. https://doi.org/10.12890/2019_001231

Rivera-Chavarría, I. J., \& Brenes-Gutiérrez, J. D. (2016). Thromboangiitis obliterans (Buerger's disease). Annals of medicine and surgery; 7, 79-82. https://doi.org/10.1016/j.amsu.2016.03.028

Sanabria, R. J. F., Herrera, A. L. G., Pereira, M. R., Rolando, I. E. C. \& Martin, O. P. (2020). Eritromelalgia. Informe de un caso. Rev Méd Electrón; 42(6). http://www.revmedicaelectronica.sld.cu/index.php/rme/article/view/3295/4971

Santana, C. L. M., Aguiar, F. S., Rosado, M. B., Watashi, D. M., Ferreira, L. V. \& Portela, L. A. (2020). Tromboangeíte obliterante com impossibilidade de revascularização: relato de caso clínico. Revista Científica UMC Mogi das Cruzes, 5(1). http://seer.umc.br/index.php/revistaumc/article/view/733/748

Suzuki, J (2018). Achenbach's Syndrome in a 30-year-old Healthy Woman. Intern Med Advance Publication. 10.2169/internalmedicine.2263-18.

Watchorn R. E., Babu S., Lewis F., Calonje E. \& Taibjee S. M. (2017). Paroxysmal purple palmar macules with a rare aetiology. Clin Exp Dermatol; 42(5):561563. 10.1111/ced.13101.

Wigley F. M. \& Flavahan N. A (2016). Raynaud's Phenomenon. New England Journal of Medicine; 11;375(6):556-65. 10.1056/NEJMra1507638

Yamada, T. (2018). Achenbach's syndrome in an elderly woman. Journal of General and Family Medicine; 00:1-2. https://doi.org/10.1002/jgf2.158.

Yie, K (2019). Achenbach Syndrome: A Benign Painful Blue Finger with Tip Sparing. Vasc Specialist Int; $\quad$ 35(4):251-253. https://doi.org/10.5758/vsi.2019.35.4.251. 